\title{
Archipel
}

ARCHIPEL Études interdisciplinaires sur le monde insulindien

$88 \mid 2014$

Varia

\section{In memoriam : Christian Pelras (1934-2014)}

\section{Pierre Labrousse}

\section{OpenEdition}

Journals

Édition électronique

URL : http://journals.openedition.org/archipel/524

DOI : 10.4000/archipel.524

ISSN : 2104-3655

\section{Éditeur}

Association Archipel

\section{Édition imprimée}

Date de publication : 10 octobre 2014

Pagination : 3-7

ISBN : 978-2-910513-71-9

ISSN : 0044-8613

\section{Référence électronique}

Pierre Labrousse, «In memoriam : Christian Pelras (1934-2014) », Archipel [En ligne], 88 | 2014, mis en ligne le 10 septembre 2017, consulté le 25 septembre 2020. URL : http://journals.openedition.org/ archipel/524 ; DOI : https://doi.org/10.4000/archipel.524 


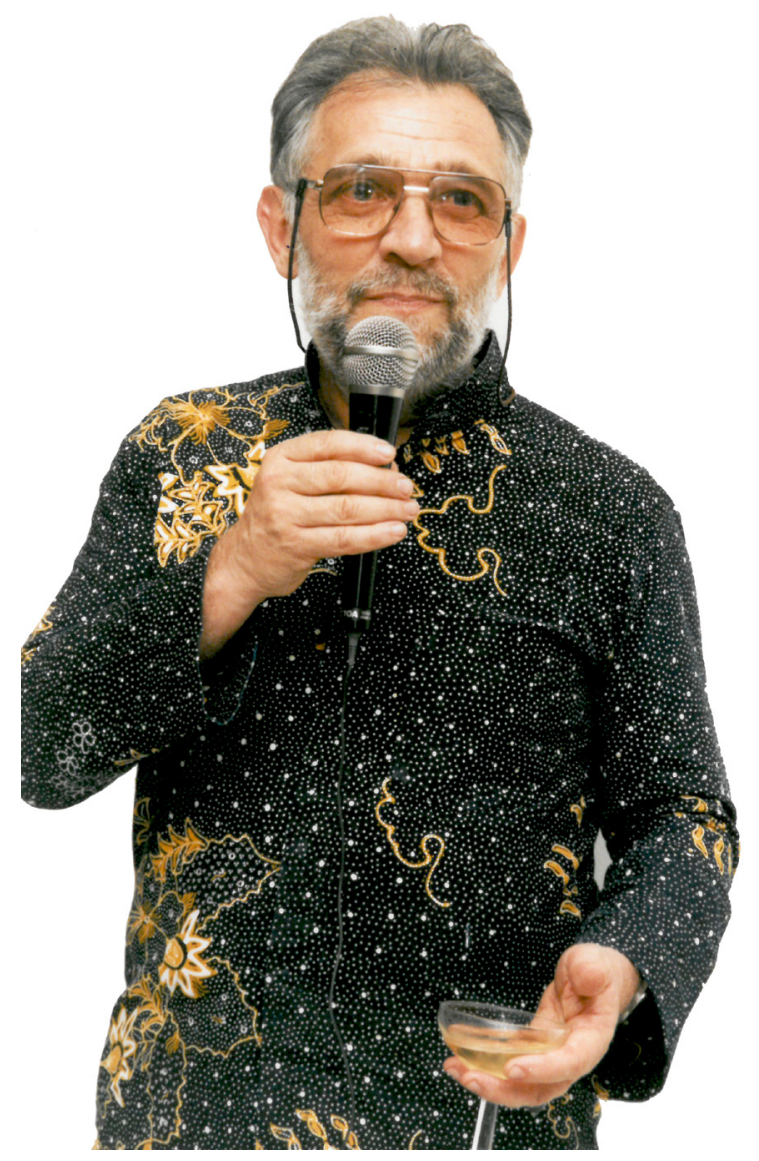

\section{Christian Pelras (1934-2014)}

Christian Pelras était l'un des fondateurs de la revue Archipel. Avec sa disparition s'arrête une longue collaboration de quarante-trois années qui correspondent à l'émergence de l'Insulinde dans la recherche française. Il était en quelque sorte le dernier des pionniers. Mais au moment d'écrire un aperçu de sa carrière, il paraît difficile de le cerner parce qu'il a été un homme aux fidélités multiples, aux activités diversifiées. Il a été attentif aux hommes, aux objets, aux techniques, arpentant le terrain de l'Alsace à la Bretagne et de Célèbes-Sud à la Malaisie, ouvert aux jeunes chercheurs et consacrant beaucoup de son temps à diverses institutions de Paris à Macassar. Il a toujours été, comme il le reconnaît lui-même, dès ses études partagées entre l'Aveyron et Paris, un peu en décalage et ailleurs ${ }^{1}$.

1. Michel Picard, Hugues Tertrais, 2001, "Christian Pelras, ethnologue planétaire et ami des Bugis », La lettre de l'AFRASE 55, p. 5-14. 
Après les curiosités de jeunesse, le Musée de l'Homme apparaît, dans ses souvenirs, au premier rang des relais qui déterminent sa vocation. C'est en tout cas ce lieu, à la fois consacré aux civilisations étrangères et aux traditions de la France rurale qu'il désigne comme l'origine de son attrait pour un au-delà de ses études classiques en hypokhâgne et khâgne à Janson-de-Sailly. À cette époque la formation en ethnologie n'était pas structurée comme aujourd'hui. Elle était organisée autour d'une licence libre que le candidat combinait en fonction de ses centres d'intérêts. Il suit les cours d'André Leroi-Gourhan qui l'accompagnera durant tous ses débuts. Il est l'élève de Georges Gurvitch, Jean Duvignaud, Jean-Pierre Faye pour la sociologie, Gabriel Legras pour la sociologie religieuse. La licence passée, il fait un stage au centre de Formation aux recherches ethnologiques qui était installé au Musée de l'Homme. Là il est initié aux techniques de terrain et aux objets pour les collections qui constitueront l'une de ses préoccupations constantes. C'est là aussi, dans le département d'Océanie, parmi les témoins des cultures matérielles, qu'il choisit de s'intéresser aux métiers à tisser sur lesquels il gardera une attention particulière tout au long de sa carrière.

Toujours dans l'analyse des vocations qui pour nos milieux sont toujours plus ou moins singulières, il est aussi redevable de son orientation à Louis Berthe ${ }^{2}$ (1927-1968), lui-même ethnologue, qui va le convaincre de s'intéresser à l'Indonésie où il a déjà fait de longs séjours depuis 1957, surtout dans la partie Est (Timor, Sumbawa, Bali et Java, chez les Baduy), puis avec une mission de l'UNESCO pour étudier les conséquences de l'éruption du Gunung Agung. Ce noyau qui comprend Claudine Berthe, Henri et Maria Campagnolo, Brigitte Clamagirand constitue la première génération des ethnologues français de l'Indonésie. Christian Pelras se situe juste quelques années après eux. En Indonésie le cadre politique est celui des débuts de l'Indépendance, avec une grande sympathie pour les nouveaux étrangers quand ils ne sont plus soupçonnés de connivences coloniales.

Dans ces années-là, l'Indonésie travaille à construire ses relations internationales inspirées par une idéologie anticolonialiste et tiers-mondiste active. Aussi quand l'Ambassade de France à Jakarta propose des bourses d'études pour des étudiants indonésiens, le Président Sukarno s'empresse de répliquer que les relations doivent fonctionner dans les deux sens et offre la réciproque, c'est-à-dire des bourses indonésiennes à des étudiants français. Les premiers à bénéficier de cette proposition furent Françoise Cayrac, Christian Pelras et un spécialiste des papillons. Cette initiative fut sans lendemain, mais au moins fut-elle déterminante pour la vocation de deux futurs chercheurs sur l'Indonésie.

C'est dans ce cadre-là qu'il se trouve d'octobre 1960 à décembre 1961, à Jakarta. Il a son quartier général à la bibliothèque du musée qui a conservé le fonds colonial et où il dispose également des collections ethnographiques. Il a toujours en tête une étude élargie sur les tissages traditionnels et en profite pour voyager à Java et à Bali.

2. Cf. la nécrologie de Louis Berthe par Christian Pelras, Archipel 1, 1971, p. 97-102. 
Au retour, il entreprend pour son troisième cycle un travail sur un village alsacien, Bitschwiller-lès-Thann où le ramènent les visites familiales chez son épouse, Marie-Thérèse. Ensuite grâce à Leroi-Gourhan, il est attaché au Museum pour un important programme interdisciplinaire en Bretagne, à Goulien (Finistère) où il a la particularité d'arriver avec un regard ethnologique formé à Jakarta. Ce projet est considérable, il recroise les recherches de plusieurs personnes et il a encore à ce jour des prolongements. Il progresse d'ailleurs si vite que la thèse que Christian Pelras pensait faire sur son village alsacien sera doublée en quelque sorte par ses recherches en Bretagne ${ }^{3}$ dont il devient l'un des ethnologues attitrés. Il restera fidèle à ces attaches ethnographiques en France, avec des contacts réguliers - retour sur terrain et bilan des changements en 1'an 2000 ${ }^{4}$. Il fera même dans certaines universités indonésiennes des conférences sur ce village français pour montrer, au grand étonnement de ses auditeurs, que la distance n'est pas si grande qu'on imagine.

En 1964 il est recruté au CNRS avec un programme qui englobe les trois références citées ci-dessus : Bitschwiller, Goulien et l'Indonésie vers laquelle l'oriente son nouveau rattachement au Cedrasemi de Georges Condominas. Il reste fidèle à ses terrains ethnologiques français, mais il va rapidement constater que l'Insulinde sollicite l'essentiel de son temps.

En arpentant la Malaisie (1967), pour son enquête sur les tissages, il a rencontré une petite communauté d'émigrés bugis. C'est par eux qu'il va entrer à Célèbes Sud, tout en gardant les relations avec la Malaisie. Il est naturellement confronté à l'immense complexité des cultures indonésiennes, à plus forte raison quand il s'agit de regarder tout autour pour ancrer des points de comparaison avec les ethnies voisines. Il suit également les Bugis, géographiquement dans leur espace de migration et historiquement dans leur littérature ou dans leur modernisation au contact des grands centres urbains indonésiens et étrangers comme Singapour. Par leur diaspora, leurs techniques navales ou leur littérature encore, les Bugis sont un excellent exemple de diffusion et de contacts.

Toujours en 1967, il fait la connaissance de Denys Lombard qui est alors en poste à l'EFEO à Jakarta. Les deux chercheurs ont des approches sensiblement différentes et Denys Lombard ne se prive pas de reprocher avec vivacité la pratique d'une « certaine anthropologie intemporelle, insuffisamment sensible au devenir réel des sociétés » ${ }^{5}$. En fait Christian Pelras est acquis à plus de dimension historique mais la question sera récurrente dans les réunions du comité de rédaction d'Archipel et le débat sans fin...

3. Goulien, commune rurale du Cap-Sizun (Finistère). Étude d'ethnologie globale, Cahiers du Centre de Recherches anthropologiques, 6, Paris, Masson, 1965.

4. Le maire de Goulien annonce son intention de donner à la bibliothèque municipale le nom de Christian Pelras.

5. Christian Pelras, Mémoire de titres et travaux, 2001, p. 7. Voir aussi « Entretien avec Christian Pelras ». Entretien réalisé par Frank Michel, Histoire et Anthropologie, n 10, janvier-juin 1995, p. $85-92$. 
En 1970 en effet, a été créée la revue Archipel. Chaque chercheur quelque peu entreprenant ou ambitieux songe à un moment de sa carrière à fonder une revue. À l'époque l'Indonésie vue de France était un espace blanc, à peine cartographié dans les esprits et politiquement agité, considéré comme une région à risque élevé, en proie à l'incertitude des balancements de Sukarno entre les deux blocs de la guerre froide. L'entreprise se justifiait pleinement. C'est Denys Lombard qui fit la jonction entre Christian Pelras et moi-même dans un café de la place du Trocadéro. Nos motivations étaient différentes mais elle se complétaient.

Cette référence à l'histoire et l'impossibilité à constituer une équipe insulindienne autonome cristallise aussi la scission du Cedrasemi (1978). Le groupe orientalisant que nous étions s'organise de façon autonome autour de la direction d'études de Denys Lombard à l'EHESS. Christian Pelras ne suit pas cette dissidence, il garde sa fidélité au Cedrasemi tout en maintenant ses activités dans 1'Association Archipel et dans la rédaction de la revue, ainsi que tous ses rapports d'amitié. En 1981, il est l'éditeur d'un volume spécial sur Célèbes-Sud (Archipel 10) qui réunit les contributions de collègues de Macassar. C'est à partir de cette région qu'il développe sa réflexion sur la technologie comparée, en particulier sur ces bases de départ que sont une typologie et une classification préalables à toute comparaison. Il prend l'exemple des métiers à tisser.

Il n'a jamais perdu de vue une autre de ses formations initiales, à savoir la question des collections ethnographiques du Musée de l'Homme qui a été le principal lieu de sa formation universitaire. L'usage pour les explorateurs en mission officielle était de rapporter des objets, de les exposer, après un rapport académique. Christian Pelras est fidèle à cette tradition, en dépit des formalités et des complications pour leur acquisition et leur sortie du territoire national. De 1961 à 1997, chacune de ces missions a donné lieu à l'envoi d'objets. Avec 1200 pièces, dont la moitié originaires de Célèbes-Sud, il se place au tout premier rang des grands contributeurs à l'enrichissement des collections. Et encore faut-il ajouter les objets de Bretagne au musée des Arts et Traditions populaires. Il s'implique progressivement dans toute la chaîne, de l'enregistrement au classement et à la description, enfin à l'exposition temporaire sur les Bugis et permanente dans les salles publiques.

Christian Pelras n'était pas non plus de ces chercheurs qui se dérobent aux activités d'enseignement. Par un certain sens de la transmission du savoir et plus profondément - nous semble-t-il — par altruisme chrétien, qualité rarement invoquée dans la recherche. Il a l'occasion de parler de la langue et littérature bugis à l'INALCO, d'ethnologie de l'Insulinde à l'EPHE ( $\mathrm{V}^{\mathrm{e}}$ section) ou encore dans les séminairesateliers du LASEMA et dans bien d'autres établissements. Ses conférences sont très nombreuses et il se prête de bonne grâce à cet exercice. Ce goût du contact se traduit également dans la vie associative. Christian Pelras a en effet co-fondé 1'AFRASE et la Société des études Euro-Asiatiques, ainsi que les « cahiers » de cette dernière.

Les responsabilités administratives ne lui manquent pas. Il est directeur de la formation du CNRS « Dynamique, Espace, Variation en Insulinde » (DEVI) 
(1985-1988), devenue LASEMA (Laboratoire Asie du Sud-Est et Monde Austronésien) dont il est le sous-directeur de 1988 à 1994.

Plus importantes, méthodologiquement parlant, sont sans doute les deux années (1978-1979) passées à l'université Hasanuddin de Macassar pour un programme doctoral à l'attention de chercheurs indonésiens. Christian Pelras se trouve au contact de toute une génération de doctorants dont les meilleurs ont soutenu leur thèse, soit sur place soit avec des compléments de formation à l'étranger, dont les Pays-Bas et la France. Si les études sur Célèbes-Sud sont aujourd'hui soutenues par un développement des chercheurs locaux et étrangers, avec des colloques, et un rayonnement international, il est certain que Christian Pelras a contribué pour une bonne part à ce développement. Enfin la publication de la synthèse sur les Bugis ${ }^{6}$ qui est une référence obligée l'a installé parmi les pères fondateurs des études sur Célèbes-Sud.

Dans la question rebattue, relative à la définition de l'ethnographie, de l'ethnologie et de l'anthropologie, Christian Pelras se réclame clairement, comme il le dit lui-même, d' " une ethnologie globale et plurithématique, — dans la lignée des travaux de Lucien Bernot et de Georges Condominas - , appuyée sur le concret vécu au niveau local, mais prenant peut-être davantage en compte les phénomènes qui se produisent au niveau des entités étatiques modernes ou des ensembles régionaux, et donnant ainsi une plus grande importance au temps long de l'histoire ${ }^{7}$.

Depuis longtemps déjà, l'association Archipel avait proposé à Christian Pelras la publication d'un recueil d'articles qu'il jugeait les plus pertinents. Il avait commencé par le projet de trois volumes dont l'édition aurait été trop longue et nous pressentions que cette ambition de départ dépassait ce que le temps lui permettait encore, physiquement, d'espérer. Avec l'aide de ses amis l'édition des Explorations dans l'univers des Bugis. Un choix de trente-trois rencontres dans les Cahiers d'Archipe ${ }^{8}$ a pu être réalisée in extremis sous son contrôle. Par " rencontres » il voulait dire que la relation fonctionnait dans les deux sens. Il serait donc heureux d'entendre que cette thématique-là lui a attiré beaucoup de nouveaux lecteurs que nous ne connaissions pas.

P.L.

6. The Bugis, Oxford, 1996. Traduction indonésienne : Manusia Bugis, Jakarta, Nalar, Forum JakartaParis, EFEO, 2010.

7. Mémoire de titres et travaux, 2001, p. 11.

8. $\mathrm{N}^{\circ} 39,2010$. 
